\title{
The basics of prescribing infant formulas
}

Owens CJW, BScDietetics; Labuschagne IL, BScDietetics; Lombard MJ, PhD Nutrition Information Centre, Stellenbosch University Correspondence to: C Owens, e-mail: nicus@sun.ac.za Keywords: infant formula, milk, whey protein, infant nutrition, feeding

\begin{abstract}
All infant formulas must support the normal growth and development of infants, and this needs to be scientifically demonstrated. Formulas have to contain sufficient amounts of basic nutrients, and so are nutritionally interchangeable, with no evidence indicating that one brand is superior to another. ${ }^{1,2}$
\end{abstract}

\section{Introduction}

Regulatory bodies around the world agree that breastfeeding provides the best nutrition for infants. ${ }^{1}$ Exclusive breastfeeding is recommended by the World Health Organization (WHO) for the first six months, and supplemental breastfeeding for up to two years, or more. Although breastfeeding provides more benefits than formula feeding, many parents still choose infant formula as an alternative to the former. ${ }^{3,4}$ Human milk contains antibodies, hormones, enzymes, and many other components, which cannot be added to formula. However, additives with proven benefits, are added to modern day infant formulas. ${ }^{5}$

This article looks at the basics of prescribing infant formula. Serious medical cases, such as short bowl syndrome and malabsorption, are beyond the scope of this article.

\section{Components of standard infant formulas}

The nutritional composition of all infant formulas must meet the global standards as recommended by the European Society for Paediatric Gastroenterology, Hepatology and Nutrition's (ESPGHAN) international expert group that was commissioned by The Codex Alimentarius Commission in November 2004. All infant formulas must contain sufficient amounts of basic nutrients, and are thus nutritionally interchangeable (Table I). No evidence exists to recommend one brand over another. ${ }^{1,2}$

\section{Prescribing the correct infant formula}

Infant formulas developed as unmodified cows' milk are not recommended for infants younger than 12 months of age, as they pose serious health risks. ${ }^{6,7}$
A wide variety of infant formulas are available, which makes choosing a suitable one difficult for parents and healthcare professionals. The majority of infants who are not being breastfed can use a standard infant formula, but a select few will require a more specialised formula. This will depend on gestational age and the presence of an illness. ${ }^{1}$

Table II provides a guide of available infant formulas, and indication of use. Formulas that are marketed for infants who are older than one year of age are not included, as no evidence exists to promote the use of such milk, or to demonstrate their superiority in terms of growth or development, when compared to whole milk. ${ }^{6}$

\section{Casein- vs. whey-predominant infant formulas}

Manufacturing companies market casein- or curdpredominant formulas to "hungry" babies. However, there is no conclusive evidence that babies sleep longer, or have better quality sleep, if given such milk. These formulas form relatively indigestible curds in the stomach, and are not recommended for young babies. ${ }^{6}$

By comparison, whey-predominant infant formulas are more easily digested. Its composition of whey:casein (60:40) is similar to that found in human milk. For this reason, wheypredominant infant formulas are usually prescribed for newborn infants. ${ }^{6}$

\section{Additional components added to infant formulas}

As more human milk components are discovered, modifications to existing infant formula and new infant formulas are made. ${ }^{5}$ The European Society for Paediatric Gastroenterology, Hepatology and Nutrition's (ESPGHAN) 
Table I: The European Society for Paediatric Gastroenterology,

Hepatology and Nutrition's recommended standards for the macro- and micronutrient composition of infant formula

\begin{tabular}{|c|c|c|}
\hline Component & Minimum & Maximum \\
\hline Energy: kcal/100 ml & 60 & 70 \\
\hline $\begin{array}{l}\text { Protein: } \mathrm{g} / 100 \mathrm{kcal} \\
\text { Cows' milk } \\
\text { Soy } \\
\text { Hydrolysed }\end{array}$ & $\begin{array}{l}1.8 \\
2.25 \\
1.8^{a}\end{array}$ & $\begin{array}{l}3 \\
3 \\
3\end{array}$ \\
\hline $\begin{array}{l}\text { Lipids: } \mathrm{g} / 100 \mathrm{kcal} \\
\text { Total fat } \\
\text { Linoleic acid } \\
\alpha \text {-linoleic acid }\end{array}$ & $\begin{array}{l}4.4 \\
0.3 \\
50\end{array}$ & $\begin{array}{c}6.0 \\
1.2 \\
\text { Not specified }\end{array}$ \\
\hline Carbohydrates: $\mathrm{g} / 100 \mathrm{kcal}$ & 9.0 & 14.0 \\
\hline $\begin{array}{l}\text { Fat-soluble vitamins: } \mu \mathrm{g} / 100 \mathrm{kcal} \text { (unless } \\
\text { otherwise specified) } \\
\text { Vitamin } \mathrm{A}: \mu \mathrm{g} \mathrm{RE} / 100 \mathrm{kcal} \text { (retinol } \\
\text { equivalent) } \\
\text { Vitamin } \mathrm{D}_{3} \\
\text { Vitamin } \mathrm{E}: \mathrm{mg} \alpha-\mathrm{TE} / 100 \mathrm{kcal} \text { ( } \alpha \text {-tocopherol } \\
\text { equivalent) } \\
\text { Vitamin } \mathrm{K}\end{array}$ & $\begin{array}{c}60 \\
1 \\
0.5 \\
4\end{array}$ & $\begin{array}{c}180 \\
2.5 \\
5 \\
25\end{array}$ \\
\hline $\begin{array}{l}\text { Water-soluble vitamins: } \mu \mathrm{g} / 100 \mathrm{kcal} \\
\text { (unless otherwise specified) } \\
\text { Thiamine } \\
\text { Riboflavin } \\
\text { Niacin } \\
\text { Vitamin } \mathrm{B}_{6} \\
\text { Vitamin } \mathrm{B}_{12} \\
\text { Pantothenic acid } \\
\text { Folic acid } \\
\text { Vitamin } \mathrm{C}: \mathrm{mg} / 100 \mathrm{kcal} \\
\text { Biotin }\end{array}$ & $\begin{array}{c}60 \\
80 \\
300 \\
35 \\
0.1 \\
400 \\
10 \\
8 \\
1.5\end{array}$ & $\begin{array}{c}300 \\
400 \\
1500 \\
175 \\
0.5 \\
2000 \\
50 \\
30 \\
7.5\end{array}$ \\
\hline $\begin{array}{l}\text { Minerals and trace elements: mg/100 } \\
\text { kcal } \\
\text { Iron (cows' milk and hydrolysate protein) } \\
\text { Iron (soy-based protein) } \\
\text { Calcium } \\
\text { Phosphorus (cows' milk and hydrolysate } \\
\text { protein) } \\
\text { Phosphorus (soy-based protein) } \\
\text { Magnesium } \\
\text { Sodium } \\
\text { Chloride } \\
\text { Potassium } \\
\text { Minerals and trace elements: } \mu \mathrm{g} / 100 \\
\text { kcal } \\
\text { Manganese } \\
\text { Fluoride } \\
\text { lodine } \\
\text { Selenium } \\
\text { Copper } \\
\text { Zinc }\end{array}$ & $\begin{array}{c}0.3^{b} \\
0.45 \\
50 \\
25 \\
30 \\
5 \\
20 \\
50 \\
60 \\
\\
1 \\
\text { Not specified } \\
10 \\
1 \\
35 \\
0.5\end{array}$ & $\begin{array}{c}1.3 \\
20 \\
140 \\
90 \\
100 \\
15 \\
60 \\
160 \\
160 \\
\\
50 \\
60 \\
50 \\
9 \\
80 \\
1.5\end{array}$ \\
\hline $\begin{array}{l}\text { Other substances: } \mathrm{mg} / 100 \mathrm{kcal} \\
\text { Choline } \\
\text { Myo-inositol } \\
\text { L-carnitine }\end{array}$ & $\begin{array}{c}7 \\
4 \\
1.2\end{array}$ & $\begin{array}{c}50 \\
40 \\
\text { Not specified }\end{array}$ \\
\hline
\end{tabular}

$a=$ If an infant formula is based on hydrolysed protein, and it contains less than $2.25 \mathrm{~g} / 100 \mathrm{kcal}$ protein, it should be clinically tested.

$\mathrm{b}=\ln$ areas where iron deficiency is proven, a higher iron content can be approved at national level.

international expert group, commissioned by The Codex Alimentarius Commission in November 2004, reported that the mere fact that a component is found in human milk, does not justify its addition to formula. Formula milk additives have to demonstrate that they provide a scientifically proven benefit. $^{2}$

\section{Long-chain polyunsaturated fatty acid supplementation}

Alpha-linolenic acid (ALA) is the precursor for docosahexaenoic acid (DHA), more commonly referred to as omega-3 fatty acids. Linoleic acid (LA) is the precursor of arachidonic acid (AA), also known as omega- 6 fatty acids. Omega- 3 and omega- 6 fatty acids are long-chain polyunsaturated fatty acids, important for neurological, cognitive, and visual development in the rapidly growing newborn. ${ }^{8}$

$D H A$ and $A A$ are present in human milk, in variable amounts. However, until recently, infant formulas only contained their precursors, LA and ALA. The addition of LA and ALA to formulas is standard, and they have to be added to all infant formulas on ESPGHAN's recommendation. ${ }^{1,2,9}$

In a recent Cochrane review, it was reported that the supplementation of DHA and $A A$ in formula milk has not shown any beneficial effect with regard to the visual, physical, and neurodevelopment of term infants. ${ }^{2}$ Further research is needed before routine supplementation of DHA and $A A$ in infant formulas can be recommended. ${ }^{2}$

\section{Pre- and probiotics}

Prebiotics, mostly in the form of indigestible oligosaccharides, are frequently added to infant formulas. Evidence that prebiotics reduce allergic disease is insufficient. Adding them is optional, providing they do not exceed $0.8 \mathrm{~g} /$ $100 \mathrm{ml}^{10,11}$

The motivation for adding probiotics to infant formula is that they might have a positive effect on digestion, with regard to the frequency and duration of gastrointestinal infections, including acute rotavirus gastroenteritis. They might reduce the risk of antibiotic-associated diarrhoea developing, as well as improve immunity. When added to infant formulas, Bifidobacterium lactis and Lactobacillus reuteri showed a significant decrease in the incidence and duration of diarrhoea caused by rotavirus. ${ }^{12}$ A systematic review conducted by ESPGHAN indicated that there is not enough evidence to recommend the addition of probiotics to infant formula. ${ }^{1}$

\section{Nucleotides}

A randomised control trial by Singhal et al found that healthy term infants fed nucleotide supplemented formulas experienced a greater weight increase and greater head circumference measurement compared to nonsupplemented formulas. ${ }^{13}$ ESPGHAN supports the addition of nucleotides to infant formula, provided that they do not exceed the maximum allowed amount of $5 \mathrm{mg} / 100 \mathrm{kcal} .{ }^{2,13,14}$ 
Table II: Infant formula available in South Africa, and indication of use

\begin{tabular}{|c|c|c|c|c|c|c|}
\hline Class & $\begin{array}{l}\text { Indication } \\
\text { of use }\end{array}$ & Brand name & $\begin{array}{l}\text { Kcal/ } \\
100 \mathrm{ml}\end{array}$ & Protein source and $\mathrm{g} / 100 \mathrm{ml}$ & $\begin{array}{l}\text { Carbohydrates } \\
\text { and } \mathrm{g} / 100 \mathrm{ml}\end{array}$ & Additives \\
\hline \multirow{10}{*}{$\begin{array}{l}\text { Standard term } \\
\text { infant formula } \\
0-6 \text { months of } \\
\text { age, but can be } \\
\text { used up to } 12 \\
\text { months of age }\end{array}$} & \multirow{10}{*}{$\begin{array}{l}\text { Healthy term } \\
\text { infants, of normal } \\
\text { birth weight }\end{array}$} & Nestlé, Nan $1^{\circledR}$ & 67 & $\begin{array}{l}\text { Demineralised whey, } 30: 70 \\
\text { whey-dominant, } 1.23\end{array}$ & Lactose, 7.47 & $\mathrm{DHA}^{\mathrm{a}}, \mathrm{AA}^{\mathrm{b}}$, and nucleotides \\
\hline & & Nestlé, Lactogen $1^{\circledast}$ & 67 & $\begin{array}{l}\text { Demineralised whey, } \\
55: 45 \text { casein-dominant, } 1.51\end{array}$ & $\begin{array}{l}\text { Lactose, maltodextrin, } \\
7.46\end{array}$ & - \\
\hline & & Pfizer, S26 $1^{\oplus}$ & 67 & $\begin{array}{c}\text { Skimmed milk } \\
\text { 40:60 whey-dominant, } 1.5\end{array}$ & Lactose, 7.2 & - \\
\hline & & Pfizer, S26 Gold $1^{\circledast}$ & 67 & $\begin{array}{l}\text { Skimmed milk, 40:60 } \\
\text { whey-dominant, } 1.5\end{array}$ & Lactose, 7.2 & $\begin{array}{l}\text { DHA, AA, and } \\
\text { nucleotides }\end{array}$ \\
\hline & & Pfizer, SMA $1^{\oplus}$ & 67 & $\begin{array}{l}\text { Skimmed milk, } 1.6 \\
80: 20 \text { casein-dominant }\end{array}$ & Lactose, 7.0 & - \\
\hline & & Aspen, Infacare $1^{\circledR}$ & 67 & $\begin{array}{l}\text { Whey protein, } 40: 60 \\
\text { whey-dominant, } 1.5\end{array}$ & Glucose, lactose, 7.5 & - \\
\hline & & $\begin{array}{l}\text { Aspen, Infacare } \\
\text { Gold } 1^{\circledR}\end{array}$ & 68 & $\begin{array}{l}\text { Whey protein, } 40: 60 \\
\text { whey-dominant, } 1.4\end{array}$ & Lactose, 7.5 & $\begin{array}{l}\text { DHA, AA, and } \\
\text { nucleotides }\end{array}$ \\
\hline & & $\begin{array}{l}\text { Aspen, Infacare } \\
\text { Nurture } 1^{\circledast}\end{array}$ & 67 & $\begin{array}{l}\text { Whey protein, } 40: 60 \\
\text { whey-dominant, } 1.5\end{array}$ & Lactose, 7.5 & $\begin{array}{c}\mathrm{DHA} \text { and } \mathrm{AA} \text {. } \\
\text { Prebiotics and nucleotides }\end{array}$ \\
\hline & & Pharmac, Novolac $1^{\circledR}$ & 66.1 & $\begin{array}{l}\text { Demineralised whey, } \\
\text { 50:50 whey:casein, } 1.5\end{array}$ & $\begin{array}{l}\text { Lactose, maltodextrin, } \\
7.5\end{array}$ & - \\
\hline & & $\begin{array}{l}\text { Abbott, Similac } \\
\text { Advance with Iron }{ }^{\circledast}\end{array}$ & 68 & Whey protein, 1.4 & Lactose, 7.3 & $\begin{array}{l}\text { DHA, AA, and } \\
\text { nucleotides }\end{array}$ \\
\hline \multirow{8}{*}{$\begin{array}{l}\text { Standard term } \\
\text { infant formula } \\
6-12 \text { months of } \\
\text { age (not to be } \\
\text { used for infants < } \\
6 \text { months of age) }\end{array}$} & \multirow[t]{8}{*}{$\begin{array}{l}\text { Healthy term infants } \\
\text { older than } 6 \text { months } \\
\text { of age }\end{array}$} & Nestlé, Nan $2^{\circledast}$ & 67 & $\begin{array}{l}\text { Demineralised whey, } \\
60: 40 \text { casein-dominant, } 2.02\end{array}$ & Lactose, 7.67 & $\begin{array}{c}\text { DHA } \\
\text { probiotics: Lactobacillus } \\
\text { GG and Bifidobacterium } \\
\text { longum }\end{array}$ \\
\hline & & Nestlé, Lactogen $2^{\circledR}$ & 67 & $\begin{array}{l}\text { Skim milk, } \\
\text { 64:35 casein-dominant, } 2.03\end{array}$ & $\begin{array}{l}\text { Lactose, maltodextrin, } \\
\text { sucrose, } 8.05\end{array}$ & $\begin{array}{c}\text { Probiotics: Lactobacillus } \\
\text { reuteri }\end{array}$ \\
\hline & & Pfizer, S26 Promil $2^{\circledR}$ & 67 & $\begin{array}{l}\text { Whey protein, } 60: 40 \\
\text { casein-dominant, } 2.2\end{array}$ & $\begin{array}{l}\text { Lactose and } \\
\text { sucrose, } 8.2\end{array}$ & Nucleotides \\
\hline & & $\begin{array}{l}\text { Pfizer, S26 Gold } \\
\text { Promil } 2^{\circledR}\end{array}$ & 67 & $\begin{array}{l}\text { Whey protein, } 60: 40 \\
\text { casein-dominant, } 2.2\end{array}$ & Lactose and sucrose & $\begin{array}{l}\text { DHA, AA, and } \\
\text { nucleotides }\end{array}$ \\
\hline & & Aspen, Infacare $2^{\circledR}$ & 66 & $\begin{array}{l}\text { Whey protein, } 65: 35 \\
\text { casein-dominant, } 2.4\end{array}$ & $\begin{array}{l}\text { Sucrose and glucose } \\
\text { syrup, } 8.6\end{array}$ & - \\
\hline & & $\begin{array}{l}\text { Aspen, Infacare } \\
\text { Gold } 2^{\circledR}\end{array}$ & 69 & $\begin{array}{l}\text { Whey protein, } 50: 50 \\
\text { casein:whey }\end{array}$ & Lactose and sucrose & $\begin{array}{l}\text { DHA, AA, and } \\
\text { nucleotides }\end{array}$ \\
\hline & & $\begin{array}{l}\text { Aspen, Infacare } \\
\text { Nuture } 2^{\circledR}\end{array}$ & 67.3 & $\begin{array}{l}\text { Skim milk, } 40: 60 \\
\text { casein-dominant }\end{array}$ & $\begin{array}{c}\text { Lactose and sucrose, } \\
8.1\end{array}$ & $\begin{array}{c}\text { DHA, AA, and } \\
\text { nucleotides. Pre-and } \\
\text { probiotics }\end{array}$ \\
\hline & & $\begin{array}{l}\text { Pharmaco, } \\
\text { Novolac } 2^{\circledR}\end{array}$ & 66 & $\begin{array}{l}\text { Skimmed milk, 80:20 } \\
\text { casein-dominant,1.6 }\end{array}$ & $\begin{array}{l}\text { Lactose and } \\
\text { maltodextrin, } 7.7\end{array}$ & - \\
\hline \multirow[t]{4}{*}{ Preterm formula } & \multirow{4}{*}{$\begin{array}{l}\text { Premature infants } \\
<37 \text { weeks } \\
\text { gestation. } \\
\text { Birth weight } \\
<2.5 \mathrm{~kg}\end{array}$} & $\begin{array}{l}\text { Nestlé, Pre nan } \\
\text { powder }^{\circledR}\end{array}$ & 80 & 30:70 whey, 2.3 & $\begin{array}{l}\text { Lactose, maltodextrin, } \\
8.57\end{array}$ & DHA, AA and nucleotides \\
\hline & & $\begin{array}{l}\text { Nestlé, Pre nan } \\
\text { ready to drink }{ }^{\circledR}\end{array}$ & 80 & $\begin{array}{c}100 \% \text { partially hydrolysed } \\
\text { whey protein, } 2.9\end{array}$ & 8.4 & DHA, AA and nucleotides \\
\hline & & $\begin{array}{c}\text { Abbott, Similac Special } \\
\text { Care }\left.24 \mathrm{Ca}\right|^{\circledR}\end{array}$ & 81 & $\begin{array}{l}\text { Skimmed milk, whey protein } \\
\text { concentrate, } 2.4\end{array}$ & $\begin{array}{l}\text { Lactose and corn syrup } \\
\text { solids } 8.4\end{array}$ & DHA and AA \\
\hline & & $\begin{array}{c}\text { Abbott, Similac Special } \\
\text { Care }\left.20 \mathrm{Ca}\right|^{\circledR}\end{array}$ & 68 & $\begin{array}{l}\text { Skimmed milk, } \\
\text { whey protein, } 2.0\end{array}$ & $\begin{array}{l}\text { Lactose and corn syrup } \\
\text { solids, } 7\end{array}$ & DHA and $\mathrm{AA}$ \\
\hline \multirow[t]{5}{*}{$\begin{array}{l}\text { Soya formula } \\
0-6 \text { months of age }\end{array}$} & \multirow{5}{*}{$\begin{array}{l}\text { Primary lactase } \\
\text { deficiency. } \\
\text { Galactosaemia. } \\
\text { Vegetarian diet. }\end{array}$} & $\begin{array}{l}\text { Abbott, Similac Isomil } \\
\text { Advance }^{\circledR}\end{array}$ & 68 & Soy protein isolate, 1.66 & $\begin{array}{l}\text { Corn syrup and } \\
\text { sucrose, } 7.0\end{array}$ & DHA and $A A$ \\
\hline & & Abbott, Isomil $1^{\circledR}$ & 68 & Soy protein isolate, 1.8 & $\begin{array}{l}\text { Corn syrup, sucrose, } \\
\text { maltose, } 6.9\end{array}$ & - \\
\hline & & Pfizer, Infasoy $1^{\circledR}$ & 67 & Soy protein isolate, 1.8 & Corn syrup and sucrose & - \\
\hline & & $\begin{array}{c}\text { Aspen, Infacare } \\
\text { soya } 1^{\circledR}\end{array}$ & 65 & Soy protein isolate, 1.8 & Corn syrup solids, 6.6 & - \\
\hline & & $\begin{array}{l}\text { Aspen, Infacare } \\
\text { Gold Soya } 1^{\circledR}\end{array}$ & 67 & Soy protein isolate & Corn syrup solids & DHA, AA, and nucleotides \\
\hline
\end{tabular}




\begin{tabular}{|c|c|c|c|c|c|c|}
\hline Class & $\begin{array}{l}\text { Indication } \\
\text { of use }\end{array}$ & Brand name & $\begin{array}{l}\mathrm{Kcal} / \\
100 \mathrm{ml}\end{array}$ & Protein source and $\mathrm{g} / 100 \mathrm{ml}$ & $\begin{array}{l}\text { Carbohydrates } \\
\text { and } \mathrm{g} / 100 \mathrm{ml}\end{array}$ & Additives \\
\hline \multirow{4}{*}{$\begin{array}{l}\text { Soya formula } \\
6-12 \text { months of } \\
\text { age }\end{array}$} & \multirow{4}{*}{$\begin{array}{l}\text { Primary lactase } \\
\text { deficiency. } \\
\text { Galactosaemia. } \\
\text { Vegetarian diet. }\end{array}$} & Abbott, Isomil $2^{\circledR}$ & 69 & Soy protein isolate, 2.3 & $\begin{array}{l}\text { Corn syrup, sucrose } \\
\text { and maltose, } 8.0\end{array}$ & - \\
\hline & & $\begin{array}{l}\text { Aspen, Infacare } \\
\text { soya } 2^{\circledR}\end{array}$ & 72 & Soy protein isolate, 2.2 & Corn syrup solids, 7.7 & - \\
\hline & & $\begin{array}{l}\text { Aspen, Infacare } \\
\text { Gold Soya } 2^{\circledR}\end{array}$ & - & Soy protein isolate & Corn syrup solids & DHA, AA, and nucleotides \\
\hline & & Pfizer, Infasoy $2^{\circledR}$ & 66 & Soy protein isolate, 2.5 & $\begin{array}{l}\text { Corn syrup and } \\
\text { sucrose, } 6.9\end{array}$ & Nucleotides \\
\hline \multirow[t]{4}{*}{$\begin{array}{l}\text { Anti-reflux } \\
\text { formula }\end{array}$} & \multirow{4}{*}{$\begin{array}{l}\text { Reflux associated } \\
\text { with failure to } \\
\text { thrive, respiratory } \\
\text { symptoms and } \\
\text { esophagitis }\end{array}$} & Nestlé, Nan AR & 67 & $\begin{array}{l}\text { Demineralised whey } \\
30: 70 \text { casein-dominant, } 1.24\end{array}$ & $\begin{array}{l}\text { Lactose, potato and } \\
\text { corn starch, } 7.7\end{array}$ & - \\
\hline & & $\begin{array}{l}\text { Pharmaco, Novolac AR } \\
1 \text { (Also AR 2) }\end{array}$ & 66 & $\begin{array}{l}\text { Skim milk, } 80: 20 \\
\text { casein-dominant } 1.7\end{array}$ & $\begin{array}{l}\text { Lactose and corn } \\
\text { starch, } 7.4\end{array}$ & $\begin{array}{l}\text { Long-chain } \\
\text { polyunsaturated fatty acids }\end{array}$ \\
\hline & & Aspen, Infacare $\mathrm{AR}^{\circledast}$ & 67 & $\begin{array}{l}\text { Skim milk, 80:20 } \\
\text { casein-dominant, } 1.6\end{array}$ & $\begin{array}{l}\text { Corn syrup solids, } \\
\text { carob bean gum }\end{array}$ & - \\
\hline & & $\begin{array}{c}\text { Aspen, Infacare Nurture } \\
\qquad \mathrm{AR}^{\circledR}\end{array}$ & 67 & $\begin{array}{l}\text { Skim milk, 80:20 } \\
\text { casein-dominant, } 1.6\end{array}$ & Corn syrup solids, 7.0 & DHA, AA, and nucleotides \\
\hline \multirow[t]{2}{*}{ Acidified formula } & \multirow{2}{*}{$\begin{array}{l}\text { Infants with an } \\
\text { increased risk } \\
\text { of developing } \\
\text { gastroenteritis }\end{array}$} & Nestlé, Nan Pelargon ${ }^{\circledast}$ & 67 & $\begin{array}{c}\text { Demineralised whey, } 50: 50 . \\
1.49\end{array}$ & $\begin{array}{l}\text { Lactose, maltodextrin, } \\
7.57\end{array}$ & $\begin{array}{l}\text { Chemically acidified with } \\
\text { lactic acid }\end{array}$ \\
\hline & & Aspen, Melegi® & 67 & $\begin{array}{l}\text { Whey protein, } 40: 60 \\
\text { whey-dominant, } 1.5\end{array}$ & $\begin{array}{l}\text { Corn syrup solids, } \\
\quad \text { lactose, } 7.5\end{array}$ & $\begin{array}{l}\text { Chemically acidified with } \\
\text { citric acid }\end{array}$ \\
\hline \multirow[t]{2}{*}{$\begin{array}{l}\text { Low-lactose or } \\
\text { lactose-free }\end{array}$} & \multirow{2}{*}{$\begin{array}{l}\text { Galactosaemia. } \\
\text { (However, not all } \\
\text { of the formulas } \\
\text { in this category } \\
\text { are suitable for } \\
\text { patients with } \\
\text { galactosaemia). } \\
\text { Acute diarrhoea. } \\
\text { Lactose } \\
\text { intolerance. }\end{array}$} & $\begin{array}{l}\text { Nestle, Nan } \\
\text { Lactose free }{ }^{\circledast}\end{array}$ & 67 & $\begin{array}{l}\text { Potassium caseinate, whey } \\
\text { protein } 40: 60 \text {, whey-dominant, } \\
1.4\end{array}$ & $\begin{array}{l}\text { Corn syrup } \\
\text { (maltodextrin), } 7.8\end{array}$ & DHA, AA, and nucleotides \\
\hline & & $\begin{array}{c}\text { Aspen, Infacare }{ }^{\circledR} \\
\text { Nurture LF } \\
\text { Not for galactosaemia. }\end{array}$ & 67 & Calcium caseinate, 1.5 & Corn syrup solids, 7.2 & $\begin{array}{l}\text { DHA and AA. } \\
\text { Prebiotics }\end{array}$ \\
\hline \multirow[t]{5}{*}{$\begin{array}{l}\text { Hydrolysed cows' } \\
\text { milk formula }\end{array}$} & $\begin{array}{l}\text { Limited research } \\
\text { on the prevention } \\
\text { of cows' milk and } \\
\text { other allergies } \\
\text { (infants 0-6 months } \\
\text { of age) }\end{array}$ & Nestlé, Nan $\mathrm{HA}^{\oplus} 1$ (new) & 67 & $\begin{array}{l}100 \% \text { partially hydrolysed whey } \\
\text { protein, } 1.51\end{array}$ & $\begin{array}{l}\text { Lactose, maltodextrin, } \\
7.56\end{array}$ & $\mathrm{DHA}$ and $\mathrm{AA}$ \\
\hline & $\begin{array}{l}\text { Limited research } \\
\text { on the prevention } \\
\text { of cows' milk and } \\
\text { other allergies } \\
\text { (infants } 6-12 \\
\text { months of age) }\end{array}$ & Nestlé, Nan HA $2^{\circledR}$ & 67 & $\begin{array}{l}100 \% \text { partially hydrolysed whey } \\
\text { protein, } 2.01\end{array}$ & Lactose, maltodextrin, 8 & $\begin{array}{c}\text { DHA and AA. } \\
\text { Probiotics: Lactobacillus } \\
\text { GG and } \\
\text { Bifidobacterium longum }\end{array}$ \\
\hline & \multirow{3}{*}{$\begin{array}{l}\text { Limited research } \\
\text { on the prevention } \\
\text { of cows' milk and } \\
\text { other allergies } \\
\text { (infants } 0-12 \\
\text { months of age) }\end{array}$} & $\begin{array}{l}\text { Abbott, Similac } \\
\text { Advanced } \mathrm{HA}^{\circledR}\end{array}$ & 71 & $26 \%$ degree of hydrolysis, 1.9 & Maltodextrin, 7.9 & Nucleotides \\
\hline & & $\begin{array}{l}\text { Pharmaco, } \\
\text { Novolac } \mathrm{HA}^{\circledast}\end{array}$ & 65 & $\begin{array}{c}16-22 \% \text { degree of hydrolysis, } \\
1.6\end{array}$ & $\begin{array}{l}\text { Lactose, starch, } \\
\text { glucose syrup, } 7.6\end{array}$ & - \\
\hline & & $\begin{array}{l}\text { Aspen, Infacare Nurture } \\
\text { HA Comfort }{ }^{\circledast}\end{array}$ & 67 & Hydrolysed whey, 1.5 & Corn syrup, lactose, 7.2 & $\begin{array}{l}\text { DHA and AA. } \\
\text { Probiotics }\end{array}$ \\
\hline \multirow[t]{4}{*}{$\begin{array}{l}\text { Extensively } \\
\text { hydrolysed } \\
\text { formula }\end{array}$} & \multirow{2}{*}{$\begin{array}{l}\text { Diagnosed cows' } \\
\text { milk allergy, and } \\
\text { other medical } \\
\text { conditions }\end{array}$} & Nestlé, Alfaré ${ }^{\otimes}$ & 70 & $\begin{array}{l}100 \% \text { whey protein extensively } \\
\text { hydrolysed ( } 80 \% \text { small } \\
\text { peptides, } 20 \% \text { free amino acids }\end{array}$ & $\begin{array}{l}\text { Maltodextrin and potato } \\
\text { starch }\end{array}$ & DHA and nucleotides \\
\hline & & $\begin{array}{l}\text { Abbott, Similac } \\
\text { Alimentum }^{\circledast}\end{array}$ & 66.7 & $\begin{array}{l}\text { Extensively hydrolysed casein, } \\
\text { supplemented with } 60 \% \text { free } \\
\text { amino acids, } 1.8\end{array}$ & $\begin{array}{l}\text { Sucrose, modified } \\
\text { tapioca, } 6.8\end{array}$ & $\mathrm{DHA}$ and $\mathrm{AA}$ \\
\hline & $\begin{array}{l}\text { Cow's milk protein } \\
\text { allergy } 0-6 \text { months }\end{array}$ & $\begin{array}{l}\text { Pharmaco, Novolac } \\
\text { Allernova }^{\circledast}\end{array}$ & 61.7 & $39 \%$ degree of hydrolysis, 1.4 & Glucose syrup. 6.7 & - \\
\hline & $\begin{array}{l}\text { Cow's milk protein } \\
\text { allergy }\end{array}$ & Nutricia, Pepticate ${ }^{\circledast}$ & 66 & $\begin{array}{l}\text { Extensively hydrolysed whey, } \\
1.6\end{array}$ & Maltodextrin, 7.1 & DHA, AA, and nucleotides \\
\hline
\end{tabular}




\begin{tabular}{|l|l|c|c|c|c|c|}
\hline Class & $\begin{array}{l}\text { Indication } \\
\text { of use }\end{array}$ & Brand name & $\begin{array}{c}\text { Kcal/ } \\
100 \mathrm{ml}\end{array}$ & Protein source and g/100 ml & $\begin{array}{c}\text { Carbohydrates } \\
\text { and g/100 ml }\end{array}$ & Additives \\
\hline $\begin{array}{l}\text { Free amino } \\
\text { acid-based } \\
\text { formula }\end{array}$ & $\begin{array}{l}\text { Highly allergic } \\
\text { infant. }\end{array}$ & Nutricia, Neocate & 71 & Free amino acids, 1.95 & Glucose, syrup, 8.1 \\
$\begin{array}{l}\text { Other conditions } \\
\text { where an elemental } \\
\text { diet may be } \\
\text { indicated. }\end{array}$ & & & & & \\
\hline $\begin{array}{l}\text { Highly allergic } \\
\text { infant. } \\
\text { Other conditions } \\
\text { where an elemental } \\
\text { diet may be } \\
\text { indicated. Children } \\
>1 \text { year of age. }\end{array}$ & $\begin{array}{l}\text { Nutricia, Neocate } \\
\text { advanced }\end{array}$ & 100 & Free amino acids, 2.5 & Glucose syrup, 14.6 \\
\hline
\end{tabular}

$\mathrm{a}=$ docosahexaenoic acid $\mathrm{b}=$ arachidonic acid

\section{Specialised formulas}

In a small percentage of cases, due to feeding intolerances, infants require specialised formulas. This should only be given on the recommendation of a dietitian or physician. ${ }^{1}$

\section{Protein-modified formula}

\section{Preventing cows' milk allergies, as well as others}

There is no evidence to support giving hydrolysed cows' milk over exclusive breast milk in an attempt to prevent allergies in infants. Limited evidence exists to support giving hypoallergenic (partially hydrolysed or extensively hydrolysed) formula vs. cows' milk formula to infants at high risk of developing allergies. ${ }^{15}$

Evidence indicates that giving soy-based formula, rather than cow's milk formula, does not reduce the risk of the development of allergies such as asthma, eczema, rhinitis, and cow's milk allergy, in later infancy and childhood. ${ }^{16,17}$

\section{Treatment of cows' milk allergies}

Where exclusive breastfeeding is not possible, extensively hydrolysed cows' milk protein formulas and amino acidbased formulas should be given to treat infants with diagnosed cows' milk protein allergies. Amino acid-based formulas should be given to infants who are highly allergic. ${ }^{18}$

\section{Treatment of colic}

The authors of a recent Cochrane review concluded that there is some evidence to promote offering hydrosylate cow's milk formula to bottle-fed infants suffering from colic. ${ }^{19}$ However, the recommendation is based on a small randomised control trial, and further research is required. Soy infant formulas should not be given to prevent or treat colic. $^{17}$

\section{Soy-based infant formula}

Cows' milk protein allergy (CMPA) is a major financially driven indication for soy formulas to be given, but giving hydrolysed cow's milk protein formula and amino acid-based formula has proven to be more effective, and should be the first option in treating CMPA. ESPAGHAN recommends that there should be a transition from hydrolysed cow's milk protein formula to soy-based formula after six months of age, as it is better tolerated by older infants. The viewpoint of the American Academy of Paediatrics (AAP) is as follows: "... for infants with documented CMPA, extensively hydrolysed protein formula should be considered, because $10-14 \%$ of these infants will also have a soy protein allergy."17

The only medical indication for giving soy infant formulas is galactosaemia and primary lactase deficiency. ESPGHAN and the AAP recommend a lactose-containing formula for realimentation, but a lactose-free formula is better tolerated in infants with secondary lactose intolerance after acute gastroenteritis. This has encouraged the consumption of isolated soy-based infant formula, but research has shown that the use of lactose-free formula is also well tolerated. ${ }^{17}$

\section{Carbohydrate-modified formula}

The major carbohydrate in breast milk and standard cows' milk formula is lactose. Strong evidence suggests that a lactose-free infant formula is effective in the dietary management of acute diarrhoea. ${ }^{17}$ Galactose and glucose make up lactose, and therefore, infants with galactosaemia require a lactose-free infant formula, and cannot be breastfed. ${ }^{20}$

\section{Acidified formula}

Infant formula can be chemically acidified through the addition of $L(+)$ lactic acid, or biologically through fermentation, which transforms lactose into lactic acid. The method of acidification does not have an effect on the growth of infants. The growth of pathogenic bacteria is inhibited through the use of an acidified formula, leading to a decreased incidence of diarrhoea in infants. ${ }^{20}$

\section{Preterm milk}

Human milk is the best source of nutrition for all infants, including infants who are born prematurely. ${ }^{21,22}$ An 
appropriate preterm formula should be prescribed by a neonatologist on an individualised basis, to premature infants who cannot be breastfed. It should be given in the early months, after birth. ${ }^{23}$ Continuous nutritional intervention post-discharge must be encouraged, and is of vital importance. ${ }^{24}$

Anti-reflux formula

Almost half of all infants are affected by symptomatic gastroesophageal reflux (GER). Uncomplicated GER usually resolves spontaneously, and needs no treatment. Infants with GER presenting with failure to thrive, and esophagitis and respiratory complications, typically need pharmacological, or other treatment. Non pharmacological treatment includes giving an infant formula that is pre-thickened with precooked corn starch or locust bean gum, which has been proven to decrease episodes of regurgitation and emesis. Pre-thickened formula is nutritionally safe, and contains adequate amounts of the necessary nutrients. ${ }^{1,14,25,26}$

Follow-on milk for infants aged six to 12 months

Giving follow-on milk was deemed "not necessary" by the World Health Assembly. The United Nations Children's Fund's (UNICEF) position is that parents can continue to give first milk until an infant reaches 12 months of age. First milk, together with complimentary foods, will provide enough nutrients to satisfy a healthy infant's nutritional needs. ${ }^{6}$

\section{Conclusion}

Breastfeeding is the best source of nutrition for most infants, and should be promoted, supported, and protected at all times. In rare cases, for example, infants with galactosaemia or primary lactase deficiency, or for any other medical reasons where the mother is unable, or unwilling, to breastfeed, it is important to select a suitable infant formula based on the infant's individual needs, and medical condition.

\section{Conflict of interest}

I declare that I have no financial or personal relationship(s) which may have inappropriately influenced me in writing this paper.

\section{References}

1. O'Connor NR. Infant formula. Am Fam Physician. 2009;79(7):565-570.

2. Koletzko B, Baker S, Cleghorn G, et al. Global standard for the composition of infant formula: recommendations of an ESPGHAN coordinated internationa expert group. J Pediatr Gastroenterol Nutr. 2005;41(5):584-599.

3. Hudara S, Mimouni F, Rachman Y, et al. Retrospective cohort study of a new infant formula during the first 6 months of life: reflections on growth curves, human milk and formula feeding. Isr Med Assoc J. 2010;12(11):676-680.

4. Le Huerou-Luron I, Blat S, Boudry G. Breast- v. formula-feeding: impacts on the digestive tract and immediate and long-term health effects. Nutr Res Rev.
2010;23(1):23-36.

5. Carver JD. Advances in nutritional modifications of infant formulas. Am J Clin Nutr. 2003;77(6):1550S-1554S.

6. The United Nations Children's Fund.The health professional's guide to: "A guide to infant formula for parents who are bottle feeding". 2010 [homepage on the Internet]. c2011. Available from: http://www.unicef.org.uk/Documents/Baby_ Friendly/Leaflets/health_professionals_guide_infant_formula.pdf

7. Wijndaele K, Lakshman R, Landsbaugh JR, et al. Determinants of early weaning and use of unmodified cow's milk in infants: a systematic review. J Am Diet Assoc. 2009;109(12):2017-2028.

8. Wright $\mathrm{K}$, Coverston $\mathrm{C}$, Tiedeman M, Abegglen JA. Formula supplemented with docosahexaenoic acid (DHA) and arachidonic acid (ARA): a critical review of the research. J Spec Pediatr Nurs. 2006;11(2):100-112.

9. Simmer K, Patole SK, Rao SC. Longchain polyunsaturated fatty acid supplementation in infants born at term [Cochrane review] In: The Cochrane Library, Issue 1, 2008. Oxford: Update Software.

10. Osborn DA, Sinn J. Formulas containing hydrolysed protein for prevention of allergy and food intolerance in infants. [Cochrane review] In: The Cochrane Library, Issue 4, 2006. Oxford: Update Software.

11. Braegger C, Chmielewska A, Decsi T, et al. Supplementation of infant formula with probiotics and/or prebiotics: a systematic review and comment by the ESPGHAN committee on nutrition. J Pediatr Gastroenterol Nutr. 2011;52(2):238-250.

12. Thomas DW, Greer FR. American Academy of Pediatrics Committee on Nutrition, American Academy of Pediatrics Section on Gastroenterology, Hepatology, and Nutrition. Probiotics and prebiotics in pediatrics. Pediatrics. 2010;126(6):1217-1231

13. Singhal A, Kennedy K, Lanigan J, et al. Dietary nucleotides and early growth in formula-fed infants: a randomized controlled trial. Pediatrics. 2010;126(4):e946-e953

14. Joeckel RJ, Phillips SK. Overview of infant and pediatric formulas. Nutr Clin Pract. 2009;24(3):356-362.

15. Osborn DA, Sinn J. Soy formula for prevention of allergy and food intolerance in infants [Cochrane review] In: The Cochrane Library, Issue 4, 2006. Oxford: Update Software.

16. Osborn DA, Sinn JK. Probiotics in infants for prevention of allergic disease and food hypersensitivity [Cochrane review] In: The Cochrane Library, Issue 4, 2007. Oxford: Update Software.

17. Vandenplas $Y$, De Greef $E$, Devreker T, Hauser B. Soy infant formula: is it that bad? Acta Paediatr. 2011;100(2):162-166

18. Dupont $\mathrm{C}$, de Boissieu $\mathrm{D}$. Treatment of severe cow's milk protein allergy using Neocate. Allerg Immunol (Paris). 2002;34(3):85-90.

19. Swadling C, Griffiths P. Is modified cow's milk formula effective in reducing the symptoms of infant colic? [Cochrane review] In: The Cochrane Library, Issue 4, 2011. Oxford: Update Software.

20. Velaphi SC, Cooper PA, Bolton KD, et al. Growth and metabolism of infants born to women infected with human immunodeficiency virus and fed acidified wheyadapted starter formulas. Nutrition. 2008;24(3):203-211.

21. Charpak N, Ruiz JG, KMC Team. Breast milk composition in a cohort of preterm infants' mothers followed in an ambulatory programme in Colombia. Acta Paediatr. 2007;96(12):1755-1759.

22. Hay WW, Thureen P. Protein for preterm infants: how much is needed? How much is enough? How much is too much? Pediatr Neonatol. 2010;51(4):198-207.

23. Fanaro S, Ballardini E, Vigi V. Different pre-term formulas for different pre-term infants. Early Hum Dev. 2010;86 Suppl 1:27-31.

24. 24. Henderson G, Fahey T, McGuire W. Nutrient-enriched formula versus standard term formula for preterm infants following hospital discharge [Cochrane review] In: The Cochrane Library, Issue 4, 2007. Oxford: Update Software.

25. 25. Vanderhoof $\mathrm{JA}$, Moran $\mathrm{JR}$, Harris $\mathrm{CL}$, et al. Efficacy of a pre-thickened infant formula: a multicenter, double-blind, randomized, placebo-controlled parallel group trial in 104 infants with symptomatic gastroesophageal reflux. Clin Pediatr (Phila). 2003;42(6):483-495.

26. 26. Moukarzel AA, Abdelnour $\mathrm{H}$, Akatcherian $\mathrm{C}$. Effects of a prethickened formula on esophageal $\mathrm{pH}$ and gastric emptying of infants with GER. J Clin Gastroenterol. 2007;41(9):823-829. 